\title{
LA DESAMORTIZACIÓN DE LAS TIERRAS DE LOS PUEBLOS (CENTRO DE MÉXICO, SIGLO XIX). BALANCE HISTORIOGRÁFICO Y FUENTES PARA SU ESTUDIO
}

\author{
Daniela Marino*
}

$\mathrm{E}$

n este artículo intentaré presentar una puesta al día de lo escrito en las últimas cinco décadas sobre una de las reformas más caras a la política económica liberal decimonónica: la anulación de la propiedad y usufructo colectivo de la tierra y la creación de un mercado libre de tierras, garantizando la propiedad individual, perfecta e irrevocable. En este marco, me ocuparé exclusivamente del proceso que afectó a las tierras en posesión de los pueblos indígenas del centro de México.

Aunque los postulados liberales pretendieron ser aplicados también a otros actores. La Iglesia, a pesar de su evidente influencia y poderío, y de movilizar amplios sectores a su favor en una guerra civil contra la facción liberal en el poder, resultó ser un objeto menos dificil para la política desamortizadora, como lo evidencia la bibliografía sobre el tema. ${ }^{1}$ La Iglesia tenía valiosas y muy notorias propiedades que fueron desamortizadas con bastante rapidez, y este proceso ha sido cuantificado también con relativa facilidad. Los latifundios en manos de propietarios civiles, en cambio, sólo fueron atacados en el papel por el ala más radical del liberalismo, que los consideraba tanto o más improductivos y retardatarios que la posesión comunal. No los enfrentó la política desamortizadora, por el contrario, fueron incrementados por la transferencia indivisa de las propiedades eclesiásticas a manos civiles.

Los pueblos, sin el poder e influencia de los grandes propietarios, tan sólo defendidos parcial y transitoriamente por algunas voces aisladas, intentaron

* Doctoranda en Historia, El Colegio de México.

1 Jan Bazant, Los bienes de la Iglesia en México (1856-1971), El Colegio de México, México, 1971; Robert Knowlton, Los bienes del clero y la Reforma mexicana, 1856-1910, FCE, México, 1985. 
retardar la aplicación de las tempranas leyes individualizadoras estatales, en ocasiones mediante su apoyo a diversas facciones en las guerras del primer medio siglo independiente. Sin embargo, fueron objeto de la abundante legislación sobre tierras expedida a partir de la Ley Lerdo, en el ámbito federal, y renovada en los estados desde ese año de 1856. Esta ley dictaminó que las tierras dejarían de pertenecer a las comunidades, o a los ayuntamientos de los que dependían, para ser parceladas y entregadas en propiedad a los individuos que detentaban la posesión o el usufructo, fueran comuneros o arrendatarios. ${ }^{2}$ Las dificultades prácticas en cumplir con dicho objetivo determinó su reiteración en sucesivas leyes desamortizadoras, tanto en el ámbito federal como en los estados, y la ampliación de su alcance, en particular durante la república restaurada y el porfiriato, mediante leyes de colonización y de tierras baldías. Esta acumulación legislativa ocasionó dificultades adicionales y no pocas contradicciones y dudas en su aplicación; la principal de las cuales concernía a la afectabilidad de los ejidos de los pueblos. Esta fuente de conflictos y litigios terminó hacia 1890, cuando el gobierno federal ordenó a los estados la desamortización inmediata de los ejidos.

La bibliografia contemporánea a los procesos desamortizadores, en particular la producida por sus críticos, nos describen un panorama agrario dominado por pocos y muy grandes terratenientes que se expandían, gracias a dicha legislación y en particular durante el porfiriato, sobre las tierras de los pueblos. Estos a su vez, acosados, empobrecidos, y muchas veces engañados por los mismos terratenientes y los oficiales del gobierno, acababan vendiendo sus tierras y engrosando el ejército de peones de las grandes haciendas. ${ }^{3}$ Esta imagen se nutría en el ataque al latifundismo de la bibliografía económica europea, pero también se veía corroborada -paradójicamente-por la estadística censal porfiriana, la cual dibujaba un paisaje rural conformado por pocas y muy grandes propiedades y más de $90 \%$ de la población agrícola laborando como peón o jornalero sin tierras. Esta información demostraba, aparentemente, el éxito avasallador de la política desamortizadora, y siguió siendo utilizada por la historiografia mexicanista -como un argumento tradicional para explicar la economía, las relaciones sociales y la propiedad rural durante el porfiriato- hasta hoy. No obstante, autores recientes han puesto en duda este panorama, dando cuenta de

${ }^{2}$ El texto completo de la ley en Luis Labastida, Colección de leyes, decretos, reglamentos, circulares, órdenes y acuerdos relativos a la desamortización de los bienes de corporaciones civiles y religiosas y a la nacionalización de los que administran las últimas, Tipografia de la Oficina Impresora de Estampillas, México, 1893, pp. 3 y ss.

${ }^{3}$ Las críticas más destacadas a las políticas agrarias del porfiriato en: Winstano Luis Orozco, Legislación y jurisprudencia sobre terrenos baldios, El Tiempo, México, 1895, 2 tomos, y Andrés Molina Enríquez, Los grandes problemas nacionales, Imprenta de A. Carranza e hijos, México, 1909. También a Daniela Marino, "El problema de la tierra y la propiedad comunal indígena en Andrés Molina Enríquez, antes y después de la revolución", Revista Iztapalapa, UAM, núm. 51, 2002, pp. 205-224. 
las erróneas clasificaciones de estructura de la propiedad y de categorías ocupacionales de los tres primeros censos nacionales en que se basa. ${ }^{4}$ Por otra parte, en las dos últimas décadas se ha desarrollado una línea de trabajos monográficos que, sin alcanzar reconstruir estadísticamente los alcances y efectos del proceso desamortizador, contribuyó de manera importante a matizar tanto la capacidad de negociación de los pueblos como las reales posibilidades coactivas de las agencias estatales para llevarlo a cabo.

Retomando el objetivo de este artículo, después de 1950 se inició una significativa producción sobre desamortización comunal, que dividí para su análisis en tres subgrupos, de acuerdo con la fecha de publicación y los enfoques y líneas temáticas predominantes: 1951-1970, 1971-1980 y 1981-2000.

Entre los autores que escribieron en las décadas de 1950 y 1960 destacan Moisés González Navarro, José Miranda y Jesús Reyes Heroles. Con un enfoque fundamentalmente político y legalista, estos historiadores parten de ubicar la desamortización agraria como un tema básico del ideario liberal, presente en los debates sobre sistemas de propiedad desde la constitución de Cádiz; si bien los dos primeros resaltan la oposición de las comunidades indígenas a esta política, ${ }^{5}$ Reyes Heroles la ubica dentro de una supuesta corriente de "liberalismo social" que a su entender surge empujada por las demandas populares. ${ }^{6}$ Sus fuentes fueron la prensa, las colecciones legislativas y los informes de gobierno, además de la bibliografia producida por los autores contemporáneos a la desamortización y a la reforma agraria, a quienes citan abundantemente. El ámbito estudiado es sobre todo el federal, aunque González Navarro publicó un artículo sobre el estado de Oaxaca. ${ }^{7}$

Estos autores, si bien reconocen y mencionan diversas estrategias asumidas por los pueblos indígenas en respuesta al proceso desamortizador -básicamente: una visible indiferencia a cumplir con la ley, la apelación a la justicia, el nombramiento de uno o varios representantes que denunciaran las tierras para seguir usufructuándolas de manera colectiva, y la oposición, incluso armada- no se

\footnotetext{
${ }^{4}$ François Xavier Guerra, México: del antiguo régimen a la revolución, FCE, México, 1995, 2 ts.; Jean Meyer, "Haciendas y ranchos, peones y campesinos en el porfiriato. Algunas falacias estadísticas", Historia Mexicana, vol. Xxxv, núm. 3, 1986, pp. 477-510; Frank Schenk, "Jornaleros y hacendados. La distribución de la propiedad de la tierra en el suroeste del Estado de México hacia 1900" en Manuel Miño Grijalva (comp.), Haciendas, pueblos y comunidades, ConaCulta, México, 1991, pp. 230-269.

${ }^{5}$ Moisés González Navarro, "Instituciones indígenas en México independiente", Memorias del Instituto Nacional Indigenista, IN, México, 1954, vol. VI, pp. 114-169; El porfiriato. La vida social, Daniel Cosío Villegas, Historia Moderna de México, Ed. Hermes, México, 1957, vol. 4; González Navarro, "Indio y propiedad en Oaxaca", Historia Mexicana, vol. vil, núm. 2, 1958, pp. 175-191, y "Tenencia de la tierra y población agrícola (1877-1960), Historia Mexicana, núm. 73, 1969, pp. 6286; José Miranda, "La propiedad comunal de la tierra y la cohesión social de los pueblos indígenas mexicano", Cuadernos Americanos, vol. cxLIX, núm. 6, 1966, pp. 168-181.

${ }^{6}$ Jesús Reyes Heroles, El liberalismo mexicano, UNAM, México, 1961, t. III, caps. VII y VIII.

${ }^{7}$ González Navarro, "Indio y propiedad en Oaxaca", op. cit.
} 
ocupan de ellas como problema de investigación. Para ellos son más importantes el debate de ideas sobre el sistema de propiedad planteado entre los liberales mexicanos y la labor legislativa, la acción del estado y, en menor medida, de las elites económicas. Las opiniones que del proceso desamortizador tuvieron actores contemporáneos a él, así como los intelectuales revolucionarios, tienen un peso muy importante en sus análisis y son los autores en quienes se apoyan y con quienes discuten. Finalmente, el uso acrítico - sin confrontar con fuentes locales- de las erradas cifras sobre distribución de la propiedad rural presentes en los tres primeros censos nacionales, les hizo considerar -tanto a ellos como a muchos otros autores, anteriores y posteriores- que las leyes de desamortización y de tierras baldías habían tenido un efecto devastador sobre la propiedad comunal, sobre todo durante el porfiriato. Así por ejemplo, Reyes Heroles, si bien nos recuerda que la tendencia a individualizar la propiedad comunal se remonta a la legislación gaditana, retoma las opiniones de manifiestos liberales -aunque críticos al porfirismo como Orozco, Molina Enríquez, Bulnes, e incluso personajes vinculados a la reforma agraria como José L. Cossío, González Roa y Covarrubias- al afirmar que ella no fue la verdadera intención de las leyes de Reforma, sino que una mala interpretación de éstas y un "abuso del procedimiento" por parte de las autoridades porfiristas afectó los ejidos y acentuó la concentración de tierras al dictar una legislación más permisiva al respecto-leyes de colonización del 15 de diciembre de 1883 y de tierras baldías del 26 de marzo de $1894 .^{8}$

El segundo periodo del que me ocuparé es el de 1971-1980, que, a diferencia de los otros dos comprende una sóla década e incluye pocos trabajos, si bien dos de ellos son libros. La menor duración de esta etapa la definí en correspondencia con los enfoques utilizados por estos autores. En 1972, Donald Fraser publicó un artículo importante en Historia Mexicana, cita obligada de todos los estudios subsiguientes sobre el tema. Este artículo es una revisión crítica de la historiografía existente sobre el tema, no tan novedoso en relación con la bibliografia reseñada para las dos décadas anteriores, pero que destaca por una hipótesis original: la continuidad existente en la política de desamortización de los pueblos indígenas en todo el periodo 1856-1911, refutando la tesis tradicional, ya reseñada, de un ataque deliberado a la propiedad comunal como parte de la "leyenda negra" del porfiriato. Por el contrario, este autor demuestra que la desamortización comunal fue, al igual que la eclesiástica, el objetivo original y totalmente consciente de Lerdo de Tejada al dictar la ley que le haría famoso y las circulares y reglamentos que le sucedieron. ${ }^{9}$

${ }^{8}$ Reyes Heroles, El liberalismo, op. cit., pp. 632-644.

${ }^{9}$ Donald J. Fraser, "La política de desamortización en las comunidades indígenas, 1856-1872", Historia Mexicana, vol. XXl, núm. 4, 1972, pp. 615-652. 
A diferencia del artículo de Fraser -que en menos espacio es más analítico y propositivo-, los libros de T. G. Powell y Jean Meyer ${ }^{10}$ abundan en juicios de valor condenatorios de la política de tierras liberal, al punto de calificarlas como "trágicas" para el campesinado indígena. Sin el suficiente respaldo documental ni estadístico, Powell concluye que las leyes desamortizadoras de la Reforma "destruyeron [...] la cohesión tradicional de las comunidades indígenas" y que Díaz "casi logró destruir a esa clase social", mientras que Meyer afirma que un efecto de la ley de desamortización fue "destruir las tierras de propiedad comunal", acelerando "la ruina de la pequeña propiedad de las comunidades", y el porfiriato "acaba de enajenar a los campesinos y [...] crea una masa creciente de desarraigados"."

Ambos comparten el interés por estudiar las respuestas campesinas a las políticas decimonónicas -si bien consideran que ellas sólo pudieron ser de rechazo violento, manifiesto en motines y levantamientos-, y se puede decir que inauguran un punto de vista pretendidamente desde abajo. La considero una pretensión, pues si bien manifiestan simpatía hacia la suerte corrida por campesinos e indígenas en este periodo de la historia mexicana, ésta se evidencia más en juicios ideológicos que en la utilización de fuentes o metodologías novedosas -habrá que esperar a los años 1980 para ver una renovación en este campo, incluso en monografias del propio Meyer. Por otra parte, no hay un real acercamiento a la política de los grupos campesinos ni a su participación -más allá de una oposición tan violenta como episódica- en la negociación de los proyectos nacionales a instrumentar, al punto que uno de ellos concluye que "las comunidades campesinas están fuera de la vida política nacional y no conocen el gobierno del estado o de la nación: se alzan para defender sus tierras y su autonomía". ${ }^{12}$ Esta tesis será revisada posteriormente, sobre todo en los años de $1990 .{ }^{13}$

No obstante estas críticas, el carácter de punto de partida de las tres obras reseñadas para este periodo es notorio en que aún hoy se citan abundantemente, dándoles el carácter de "clásicos". Tan es así, que los autores de la generación anterior, pese a haber escrito trabajos importantes sobre el tema son mucho menos citados.

La tercera generación estudiada comprende las obras publicadas en los años 1981-2000. Es el periodo de mayor producción historiográfica sobre el

10 Jean Meyer, Problemas campesinos y revueltas agrarias (1821-1910), SepSetentas, México, 1973; T. G. Powell, El liberalismo y el campesinado en el centro de México (1850 a 1876), SepSetentas, México, 1974. y $32-33$.

${ }^{11}$ Powell, El liberalismo, op. cit., pp. 74 y 155; Meyer, Problemas campesinos, op. cit., pp. 68

12 Meyer, Problemas campesinos, op. cit., p. 31.

${ }^{13}$ Por autores como Alicia Hernández Chávez, La tradición republicana del buen gobierno, $\mathrm{El}$ Colegio de México/FCE, México, 1993; Florencia Mallon, Peasant and Nation. The Making of Postcolonial Mexico and Peru, University of California Press, Berkeley, 1995; Peter Guardino, Peasant, Politics, and the Formation of Mexic's National State. Guerrero, 1800-1857, Stanford University Press, Stanford, 1996; Guy Thomson, ver infra. 
tema de la desamortización comunal y se caracteriza por la aparición de una fuerte tendencia hacia las monografías sobre una región, un estado, un distrito o incluso un municipio, donde se ponen a prueba los supuestos que las generaciones anteriores definieran en el plano nacional. Las regiones más estudiadas han sido las Huastecas ${ }^{14}$ y el Estado de México, ${ }^{15}$ remarcando el cuerpo jurídico estatal sobre desamortización promulgado con anterioridad a 1856 y su mayor peso en el ámbito local en relación con la legislación y los cambios políticos producidos en la capital del país. ${ }^{16}$ Esto se relaciona directamente con la

${ }^{14}$ Donald Stevens, "Agrarian Policy and Instability in Porfirian Mexico", The Americas, vol. XXXIX, núm. 2, 1982, pp. 153-166; Michael Ducey, "Tierras comunales y rebeliones en el norte de Veracruz antes del porfiriato, 1821-1880: el proyecto liberal frustrado", Anuario, Universidad Veracruzana, núm. VI, 1989, y "Liberal Theory and Peasant Practice. Land and Power in Northern Veracruz, Mexico, 1826-1900" en Robert H. Jackson (comp.), Liberals, the Church, and Indian Peasants. Corporate Lands and the Challenge of Reform in Nineteenth-Century Spanish America, University of New Mexico Press, Albuquerque, 1997, pp. 65-93; Antonio Escobar Ohmstede, "Los condueñazgos indígenas en las huastecas hidalguense y veracruzana: ¿Defensa del espacio comunal?", en Escobar Ohmstede (coord.), Indio, nación y comunidad en el México del siglo XIX, CEMCA/CIESAS, México, 1993, pp. 171-188; A. Escobar Ohmstede y Frans J. Schryer, "Las sociedades agrarias en el norte de Hidalgo, 1856-1900", Estudios Mexicanos, vol. 8, núm. 1, 1992, pp. 1-21; A. Escobar Ohmstede y Jacqueline Gordillo, "¿Defensa o despojo? Territorialidad indigena en las Huastecas, 1856-1930", Estudios campesinos en el Archivo General Agrario, CIESAS/SRA, México, 1998, pp. 17-74.

${ }^{15}$ Margarita Menegus Bornemann, "Ocoyoacac - una comunidad agraria en el siglo XIX", Historia Mexicana, vol. xxx, núm. 1, 1980, pp. 33-78, y "La desamortización de bienes comunales y municipales en el valle de Toluca (1800-1854)" en P. Iracheta y D. Birrichaga (comps.), A la sombra de la primera república federal. El Estado de México, 1824-1835, El Colegio Mexiquense, Toluca, 1999, pp. 279-297; John Tutino, "Cambio social agrario y rebelión campesina en el México decimonónico: el caso de Chalco" en Friedrich Katz (comp.), Revuelta, rebelión y revolución. La lucha rural en México del siglo XVI al siglo XX, Era, México, 1990, t. 1, pp. 94-134; Romana Falcón, "Jefes políticos y rebeliones campesinas: uso y abuso del poder en el Estado de México" en Jaime Rodríguez O. (comp.), Patterns of Contention in Mexican History, University of California/SR Books, Wilmington, 1992, pp. 243-273; Rina Ortiz Peralta, "Inexistentes por decreto: disposiciones legislativas sobre los pueblos de indios en el siglo XIX. El caso de Hidalgo" en Escobar Ohmstede (coord.), Indio, nación, op. cit., pp. 153-169; Frank Schenk, "La desamortización de las tierras comunales en el Estado de México (1856-1911). El caso del distrito de Sultepec", Historia Mexicana, núm. 177, 1995, pp. 3-37.

${ }^{16}$ Este tema ya había sido señalado por Moisés González Navarro, "Instituciones, indígenas", op. cit., y en Anatomia del poder en México, 1848-1853, El Colegio de México, México, 1977, cap. 3, y analizó el caso particular de Oaxaca en González Navarro, "Indio y propiedad", op. cit. Ver también Robert Knowlton, "La individualización de la propiedad corporativa civil en el siglo XIX: notas sobre Jalisco", Historia Mexicana, vol. xxviII, núm. 1, 1978, pp. 24-61, y "La división de las tierras de los pueblos durante el siglo XIX: el caso de Michoacán", Historia Mexicana, vol. XL, núm. 1, 1990, pp. 3-25; Jean Meyer, "La desamortización de las comunidades en Jalisco" y "La desamortización de 1856 en Tepic" en Meyer, Esperando a Lozada, El Colegio de Michoacán/ ConACYT, México, 1984, pp. 111-139 y 141-170, respectivamente. Para el caso de Veracruz véase Ducey, "Tierras comunales y rebeliones", op. cit., y "Liberal Theory", op. cit., Escobar y Gordillo, "¿Defensa o despojo?, op. cit. De los casos analizados, el Estado de México parece ser la excepción, pues a partir del debate en el constituyente de 1824 sobre si desamortizar o municipalizar las propiedades de los pueblos, se prefirió legislar sobre la segunda opción, de manera de garantizar recursos a los ayuntamientos por medio de la dotación de propios. Siguiendo la política central, es a partir de 1856 que se legislará sobre desamortización comunal en el Estado de México, ver Charles Hale, El liberalismo mexicano en la época de Mora, Siglo XXI, México, 1972. pp. 233 y ss.; Ortiz Peralta, "Inexistentes por decreto", op. cit.; Menegus Bornemann, "La desamortización de bienes comunales", op. cit. 
utilización preferente de los archivos municipales y estatales, además de otras fuentes novedosas como el archivo de Porfirio Díaz, ${ }^{17}$ los archivos de la reforma agraria, las memorias de la Secretaría de Fomento ${ }^{18}$ y el Semanario Judicial de la Federación. ${ }^{19}$

Persiste como tema la respuesta del campesinado a las políticas de los gobiernos liberales y se consolida el concepto de "resistencia" pero aplicado en diversos contextos; prestando menos atención a los levantamientos armados, o bien situándolos como extremo de ese continuo conformado por las diferentes prácticas de resistencia, no todas violentas ni de oposición abierta. ${ }^{20}$

Otro tema que se intenta resaltar es el papel que jugaron las autoridades intermedias al facilitar o entorpecer la marcha del proceso desamortizador, operando como bisagra entre los pueblos y el gobierno estatal. Para Schenk, la figura medular fue el juez auxiliar; Falcón, así como Escobar y Schryer, acentúan el rol del jefe político, en particular a partir de la legislación de $1868 .{ }^{21}$ En cambio, Guy Thomson, en los pueblos de la Sierra norte de Puebla, al igual que Ducey en el norte de Veracruz, resaltan el papel decisivo de los gobernadores indígenas. Estos últimos cobraron una dimensión particular en los casos en que también dirigieron destacamentos de la Guardia Nacional, conformados por sus comuneros en armas, en apoyo de la causa liberal contra los conservadores y contra la intervención extranjera. En los casos en que así sucedió, la fuerza militar les dio un plus de autoridad para negociar, a favor de sus pueblos, la aplicación de las reformas sobre propiedad de la tierra. ${ }^{22}$ La capacidad de negociar la instrumentación de las reformas se hace evidente en los estudios

${ }^{17}$ Stevens, "Agrarian Policy", op. cit.

${ }^{18}$ Robert Knowlton, "El ejido mexicano en el siglo XIX", Historia Mexicana, vol. XLVIII, núm. 1, 1998, pp. 71-96.

${ }^{19}$ Robert Knowlton, "Tribunales federales y terrenos rurales en el México del siglo xIX: el Semanario Judicial de la Federación", Historia Mexicana, vol. XLVI, núm. 1, 1996, pp. 71-98.

${ }^{20}$ Guy P. C. Thomson, "Agrarian Conflict in the Municipality of Cuetzalan (sierra de Puebla): The Rise and Fall of 'Pala' Agustin Dieguillo, 1861-1894", Hispanic American Historical Review, vol. 71, núm. 2, 1991, pp. 205-258; Schenk, "La desamortización de las tierras comunales", op. cit.; Leticia Reina, "La respuesta rural en México frente al proyecto liberal modernizador del siglo XIX", en Andrés Guerrero y Heraclio Bonilla (eds.), Los pueblos campesinos de las Américas. Etnicidad, cultura e historia en el siglo XIX, Universidad Industrial de Santander, Bucaramanga, 1996, pp. 259279; Ducey, "Liberal Theory", op. cit.; Romana Falcón, "Límites, resistencias y rompimiento del orden" en R. Falcón y Raymond Buve (comps.), Don Porfirio presidente..., nunca omnipotente. Hallazgos, reflexiones y debates, Universidad Iberoamericana, México, 1998, pp. 385-406; Jennie Purnell, "With All Due Respect: Popular Resistance to the Privatization of Communal Lands in Nineteenth-Century Michoacán”, Latin American Research Review, vol. 34, núm. 1, 1999, pp. 85-121.

${ }^{21}$ Schenk, "La desamortización de las tierras comunales", op. cit.; Falcón, "Jefes políticos y rebeliones campesinas", op. cit; ; Escobar y Schryer, "Las sociedades agrarias", op. cit.

${ }^{22}$ Guy P. C. Thomson, "Agrarian Conflict", op. cit., y "La 'bocasierra': ¿cuna del liberalismo? Tres municipios serranos entre 1855 y 1889 " en A. Annino y R. Buve (coords.), El liberalismo en México, Asociación de Historiadores Latinoamericanistas Europeos, Munster, 1993, pp. 121-141 (Cuadernos de Historia Latinoamericana, núm. 1); Ducey, "Tierras comunales y rebeliones", op. cit. y "Liberal Theory", op. cit. 
sobre las Huastecas, los que nos describen la conformación de "condueñazgos" o "sociedades agrarias" por medio de las cuales las comunidades, en convivencia con las autoridades de distrito, desamortizaron las tierras de los pueblos por grandes lotes que siguieron explotándose de manera colectiva.

Lo importante a destacar de estos trabajos, en relación con los de las dos generaciones anteriores, es el papel importantísimo de la negociación entre todos los actores involucrados para determinar el éxito, los tiempos y además la forma en que iba a concretarse, o no, la desamortización, más allá de lo dictado en principio por la legislación y de los brotes violentos como último recurso. Esto evidencia además la importancia que conservaban los pueblos como actores políticos, más allá de la disolución de los sujetos colectivos deseada y legislada por las elites liberales. En este sentido, Knowlton resalta el papel jugado por los tribunales federales como instancia de apelación, demostrando, al mismo tiempo, las contradicciones entre legislación estatal y federal, resquicio por el que seguían colándose las demandas de los pueblos pese a la quita de su personalidad jurídica. ${ }^{23}$

En los estudios de esta generación se perfilan dos corrientes: una que ratifica la "leyenda negra" del porfiriato (Thomson, Escobar, Escobar y Schryer, Tutino) que, como vimos, fue construida por los críticos contemporáneos al régimen y continuó, con distinta intensidad ideológica y eficacia argumentativa, a lo largo de las tres generaciones historiográficas aquí descritas, y otra que podríamos llamar "revisionista" (Stevens, Falcón, Knowlton), en tanto relativiza dicha visión del porfiriato como el periodo de la historia independiente en que más se atacó -o con mayor efectividad - a la propiedad comunal. En el caso de los autores nombrados en primer término, se corrobora, luego de una investigación seria sobre una región acotada, que pese a la reiteración de leyes desamortizadoras a lo largo del siglo XIX, los pueblos lograron instrumentar estrategias destinadas a mantener el usufructo e incluso la propiedad colectiva de sus tierras, en muchos casos con sostenido éxito, hasta la restauración de la república o bien hasta la ascención de Díaz. Fue entonces durante el porfiriato, si bien los autores argumentan razones diferentes a la mera voluntad política, que la desamortización comunal alcanzó sus mayores éxitos. Los estudios que aquí denominamos revisionistas, igualmente bien documentados, no refutan esta conclusión general. Sin embargo, destacan casos particulares en los que observan la voluntad de los agentes del poder porfiriano en negociar con los distintos actores para llevar adelante la desamortización, manteniendo a la vez la estabilidad y el orden, lo que redundó en condiciones más favorables para los pueblos involucrados.

Más allá de este punto de vista, surgen de la bibliografía analizada dos hitos en el proceso por lograr la individualización de la propiedad de los pueblos

${ }^{23}$ Robert Knowlton, "Tribunales federales", op. cit. 
del centro de México. El primero es 1868, cuando en el Estado de México -que aún incluía Morelos e Hidalgo- pero también en Puebla, Tlaxcala, Michoacán, el D. F. y quizás otros, se dio un impulso importante al proceso privatizador, en el primer caso, por medio de la acción de los jefes políticos. Luego, 1888-1889 parece ser otro momento importante, cuando el gobierno federal circuló instrucciones a los estados para desamortizar los ejidos de los pueblos, acabando con las contradicciones de la legislación anterior y las dudas de los oficiales del gobierno.

En cuanto al objetivo de uniformar los diversos títulos de tenencia mediante la elaboración de un registro nacional de propiedad, el paso más importante parece haber sido la ley federal del 26 de marzo de 1894, dirigida a corregir la ley anterior de baldíos de 1883, ampliando la denuncia a los terrenos ocupados ilegalmente o con títulos precarios. Esta suerte de composición buscaba privatizar la mayor parte posible del territorio nacional, así como acabar con la diversidad de derechos y sistemas de propiedad de la tierra. Toda propiedad inscrita en dicho registro sería considerada a partir de entonces por el gobierno federal como "perfecta, irrevocable y exenta de toda suerte de revisión". El objetivo del registro era "constituir definitivamente una propiedad moderna [e] individual", lo que dejaba evidentemente fuera a los pueblos. ${ }^{24} \mathrm{Sin}$ embargo, la influencia que habría de tener la legislación en los procesos locales de desamortización dependía también de otros factores, que señalo al final de este artículo.

Podemos concluir que la generación 1951-1970 dejó como principal aporte su análisis de la legislación federal y de los debates intraelite sobre el tema pero, con base en esa información, construyó una descripción desoladora del campo mexicano que encontraba ratificada en las estadísticas censales porfirianas. Ésta se vio reforzada por la imagen, muy ideologizada, que nos legó la generación 1971-1980: la de un estado porfiriano cotidianamente empeñado en favorecer el latifundismo y de disolver unas comunidades indígenas ni interesadas ni capaces de negociar con los agentes estatales, a lo sumo de estallar violentamente contra notorias injusticias, para a su vez ser reprimidas con lujo de violencia. La generación 1981-2000, mediante estudios regionales o subregionales, fue capaz de superar y complejizar este panorama lineal, fundamentalmente vía la exploración de las estrategias de negociación y conciliación de los diferentes actores involucrados. Esto, a su vez, permitió relativizar la capacidad de coacción de los centros de gobierno así como la indefensión de los pueblos indígenas, para construir en cambio una imagen donde todos los actores participaron -con diferentes capacidades según las coyunturas- en definir

${ }^{24}$ Guerra, México, op. cit., t. 1, pp. 286-289; González Navarro, El porfiriato. op. cit., pp. 189191; Menegus Bornemann, "Ocoyacac”, op. cit., p. 43; Escobar y Gordillo, “Defensa o despojo?, op. cit. 
los alcances, los tiempos y las maneras en que, en cada caso particular, se llevó a cabo el proceso desamortizador. Al respecto, la principal tarea pendiente que observo es intentar cuantificar, igualmente por medio de estudios monográficos, el real alcance de los procesos desamortizadores, así como los efectos que tuvieron para la economía campesina.

Como adelanté al comenzar este artículo, la gran mayoría de los trabajos no han logrado cuantificar los cambios en los patrones de tenencia de la tierra. Esto coincide con una de las principales armas de los pueblos y uno de los puntos más débiles en el plan desamortizador del estado durante casi todo el siglo XIX: la falta de datos confiables sobre población y propiedad que hicieron depender a los gobiernos de la buena voluntad de los pueblos y del apoyo de las autoridades intermedias para llevar adelante el reconocimiento, mensura y división de las tierras de las comunidades, así como el cobro de las cuotas e impuestos respectivos. El desarrollo de la cartografía, la estadística y el catastro corrió aunado al que experimentarían la burocracia y el ejército, a la extensión del telégrafo y el ferrocarril -que posibilitarían una más rápida intervención del centro en los ámbitos locales-y a la penetración del capitalismo en el campo, determinando todos ellos el alcance real de la desamortización en las diversas regiones y pueblos. ${ }^{25}$

De todos modos, debido a la escasez, fragmentación y poca confiabilidad de los datos recabados a lo largo de casi todo el siglo XIX, habría que remontarse

${ }^{25}$ Una primera versión de este artículo, más extensa y detallada, fue presentada como ponencia en el Primer Congreso Nacional de Historia Económica de México, que tuvo lugar en la ciudad de México del 24 al 26 de octubre de 2001. Agradezco los comentarios que me hiciera en esa ocasión Emilio Kouri, así como la referencia a su tesis doctoral, "The Business of Land: Agrarian Tenure and Enterprise in Papantla, Mexico, 1800-1910", tesis de doctorado, Dept. of History-Harvard University, septiembre 1996. Para el caso del municipio de Papantla, durante el boom de la vainilla, Kouri logra reconstruir estadísticamente la transformación de los terrenos comunales en condueñazgos, en las décadas de 1870 y 1880 , y finalmente su desamortización en lotes individuales, en los años 1890 (ver especialmente caps. 5 y 6). Agradezco igualmente a Horacio Crespo por hacerme conocer, en la misma oportunidad, su artículo "Los pueblos de Morelos. La comunidad agraria, la desamortización liberal en Morelos y una fuente para el estudio de la diferenciación social campesina", en Laura Espejel López (coord.), Estudios sobre el zapatismo, INAH, México, 2000, pp. 57-120; donde señala la existencia de una fuente privilegiada para el estudio del estado de la propiedad en el estado de Morelos en 1909, ya muy avanzado el proceso desamortizador: 50 tomos de manifestaciones prediales producto de la Ley de Revalúo General de la Propiedad Raíz dictada por el Congreso morelense en dicho año. Coincidentemente, ambos trabajos parecen indicar que en las regiones donde se desarrolló rápidamente un cultivo exportable -generalmente a fines del siglo XIX-, los procesos desamortizadores también se completaron con inaudita celeridad; de modo que el grado de penetración capitalista se convirtió en un factor mucho más importante para explicar estos procesos que la legislación emitida o la fuerza de los aparatos estatales para hacerla cumplir. Por el contrario, en zonas donde perduró la economía tradicional, hubo mayores probabilidades de que se conservara el usufructo comunal de los recursos. Ver Edgar Mendoza, "Distrito político y desamortización: resistencia y reparto de la propiedad comunal en los pueblos de Cuicatlán y Coixtlahuaca 1856-1900" y Gloria Camacho, "Resistencias cotidianas ante la intervención estatal o federal. Dos motines en torno al manejo de los recursos hidráulicos en el Estado de México. 1856-1940", ambos en Romana Falcón (comp.), La negociación del dominio, en preparación. 
hasta las últimas estadísticas borbónicas de población y catastro para una región particular y llegar hasta la información levantada para instrumentar la reforma agraria y restitución de ejidos, incorporando entonces sí los datos que puedan recogerse para los más de cien años intermedios, a través de la información de catastro, población y adjudicaciones vía desamortización en archivos municipales y estatales, así como en las oficinas del Registro Público de la Propiedad y notarías, sin descuidar los archivos judiciales. Considero que de la comparación de todos esos relevamientos es que se podrá empezar a reconstruir, aproximadamente, el impacto del proceso desamortizador en algunas áreas limitadas, que posibilite luego la elaboración de un balance más general. 


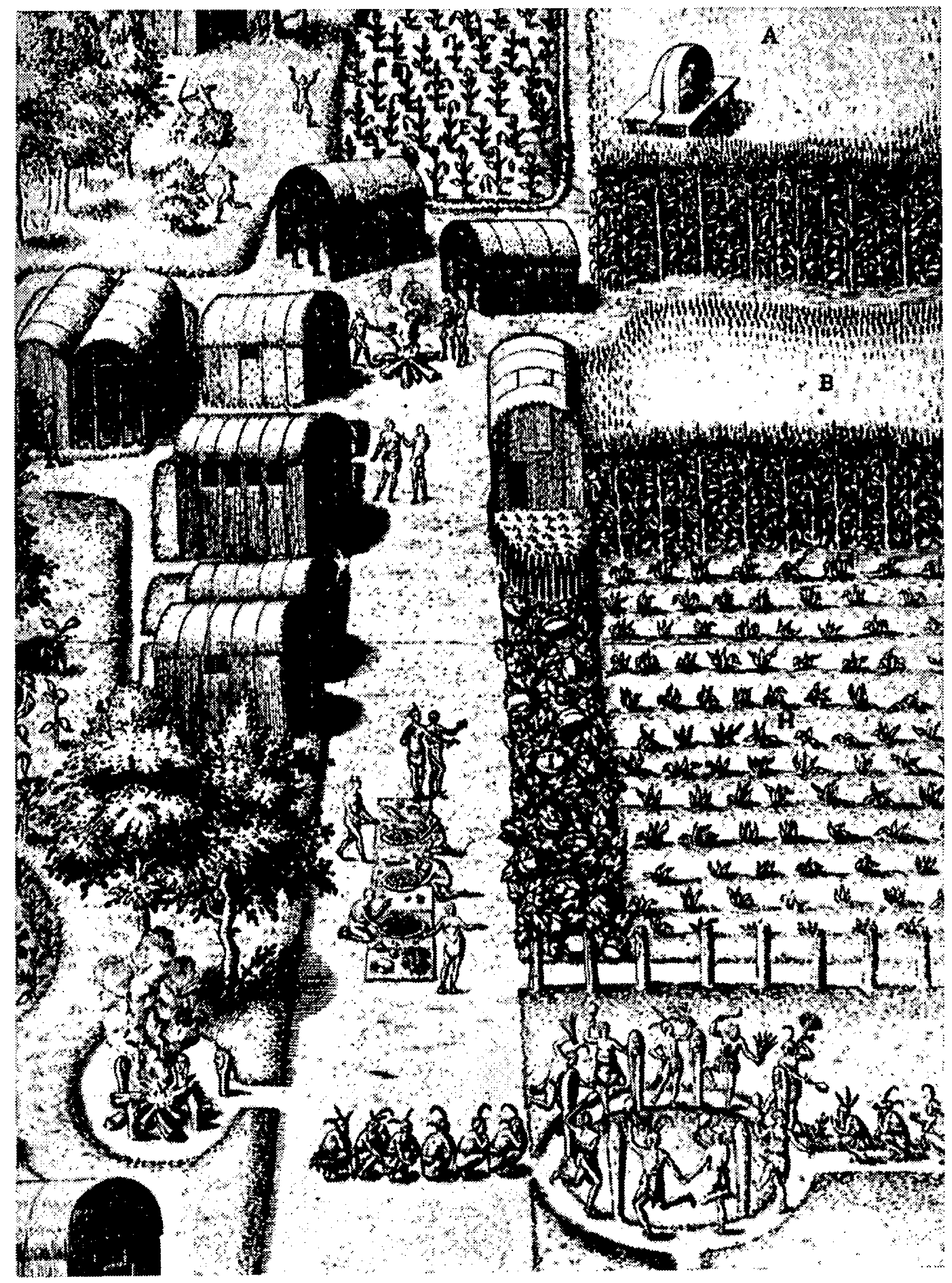

Tomado de: Howard T. Walden, Native inheritance: the story of corn in America, Harper \& Row, Publishers, Nueva York/Londres, 1966. 